\title{
Verläufe von Arbeitslosigkeit und ihre Folgen für die Wohlfahrt von Haushalten und Individuen
}

\section{Detlef Landua}

Wissenschaftszentrum Berlin für Sozialforschung, Arbeitsgruppe Sozialberichterstattung des Präsidenten, Reichpietschufer 50, D-1000 Berlin 30

Zusammenfassung: Ausgehend von einer allgemeinen Darstellung der Entwicklung der Arbeitslosigkeit in der Bundesrepublik werden mittels der Daten des „Sozio-ökonomischen Panel“ Verlaufsformen und Typen von Arbeitslosigkeit betrachtet. Arbeitslosigkeit wird nicht nur als Einschnitt individueller Lebensverläufe verstanden, sondern primär in der Konstellation eines Haushalts mit seinen einzelnen Mitgliedern. Im Rahmen dieser Kontextanalyse zeigt sich, daß haushaltsspezifische Typen von Arbeitslosigkeit bzw. von Arbeitslosen identifizierbar sind, die sich durch unterschiedliche Verlaufsformen kennzeichnen lassen. Auch die Folgen der Arbeitslosigkeit (Einkommenseinbußen, Zufriedenheitsdefizite) gestalten sich nicht einheitlich, sondern sind konkret darauf zu beziehen, welche Personen innerhalb eines Haushalts arbeitslos werden und welche Kompensationsmöglichkeiten sich der Haushaltsgemeinschaft bieten. Die Resultate weisen insgesamt auf die Notwendigkeit einer nach bestimmten Typen bzw. Verlaufsformen unterschiedenen Betrachtung des Arbeitslosenproblems hin und bestätigen die Vorteilhaftigkeit eines haushaltsbezogenen Untersuchungskonzepts.

\section{Einleitung}

In der Bundesrepublik hat sich die Zahl der Arbeitslosen in zwei Schüben auf den gegenwärtigen Jahresdurchschnitt von über zwei Millionen erhöht: Aus dem Zustand der Vollbeschäftigung wurde nach 1974 zunächst die Einmillionenschwelle, nach 1982 sodann die Zweimillionenschwelle überschritten. Seit sieben Jahren scheint diese hohe Jahresdurchschnittszahl im Aggregat unveränderlich zu sein, obwohl sich dahinter massenhafte Bewegungen ereignen. So finden wir z. B. allein im Jahre 1987 ca. 1,9 Millionen Fälle von Eintritten in die Erwerbstätigkeit aus Arbeitslosigkeit, ,Stiller Reserve" und Berufsausbildung sowie ca. 2 Millionen Austritte aus der Erwerbstätigkeit in die Arbeitslosigkeit und „Stille Reserve“. Von 1983 bis 1987 hat sich die Zahl der Arbeitsplätze um eine Million erhöht, ohne daß an der hohen Arbeitslosigkeit sich Wesentliches geändert hätte. In derselben Zeit hat sich der Anteil der „Langzeitarbeitslosen" (über ein Jahr Arbeitslosen) von 530 Tsd. auf 730 Tsd. deutlich gesteigert. Andererseits zeigen Umfrageergebnisse, daß die Angst, in der nächsten Zeit arbeitslos zu werden oder die Stelle wechseln zu müssen, die ohnehin nur eine kleine Minderheit der Erwerbstätigen äußert, von 1984 auf 1988 von $9 \%$ auf $5 \%$ bzw. von $6 \%$ auf $5 \%$ zurückgegangen ist ${ }^{1}$. Die heutige Arbeitslosigkeit ist offenbar ein komplexeres Problem als noch in der unmittelbaren Nachkriegszeit oder gar in der Weltwirtschaftskrise. Ein deutlicher wirtschaftlicher Aufschwung vermag den hohen Sockel von Arbeitslosigkeit nicht zu verringern. Langjährige
Massenarbeitslosigkeit führt nicht zu massenhafter Krisenstimmung. Subtile Ausleseprozesse vergröBern die Gruppe der langfristig oder immer wieder von Arbeitslosigkeit betroffenen Personen, während die Mehrheit sich nur wenig betroffen zeigt und kürzere Perioden der Arbeitslosigkeit inzwischen als normale Lebensereignisse wahrnimmt oder einplant. In dieser Situation sind alle Forschungsansätze von besonderem Interesse, die neue theoretische oder empirische Einsichten in das Phänomen der heutigen Arbeitslosigkeit versprechen.

Das Anliegen dieses Beitrages ist es, Arbeitslosigkeit nicht nur als Einschnitt individueller Lebensverläufe aufzufassen, sondern in der Konstellation eines Haushalts mit seinen einzelnen Mitgliedern, z.B. nach der Zahl weiterer Erwerbstätiger in diesem Haushalt. Es erscheint zumindest anzweifelbar, ob die Analyse von Arbeitslosigkeitsverläufen allein aus der Sicht der betroffenen Individuen

\footnotetext{
${ }^{1}$ Für die Grunddaten der Erwerbstätigkeit in der Bundesrepublik vgl.: Institut für Arbeitsmarkt- und Berufsforschung 1988. Zum Zuwachs der Arbeitsplätze vgl. Globus Kartendienst GmbH 1988. Die Daten über die Wahrnehmung der Arbeitsmarktsituation stammen aus den Wohlfahrtssurveys des Sonderforschungsbereichs 3, Frankfurt/Mannheim; vgl. Statistisches Bundesamt 1985, insbes. S. 410-417. Die Daten für 1988 sind noch unveröffentlicht. Zur weiteren Literatur vgl.: Franke/Prast 1985; Kreutz/Fröhlich/Maly1984; Hofbauer/Dadzio 1984; Rothe 1983; Brandes/Weise 1980: Kap. 2 und 3; Lederer 1981.
} 
- Haushaltsvorstände (1984: $N=182$ ) -
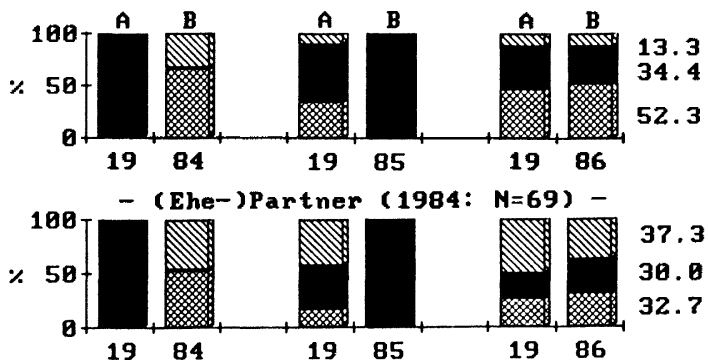

37.3

30.8

32.7

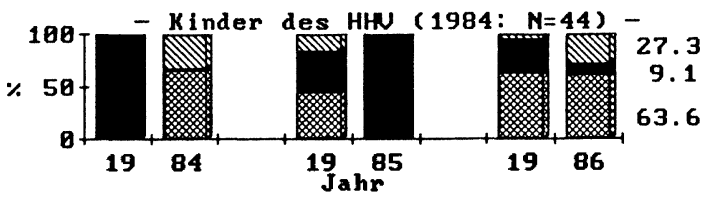

Verlaufsgruppen:

A = Gruppe 1: Arbeitslos gemeldet in 1984

B = Gruppe 2: Neu arbeitslos in 1985.

\section{Enwerbsstatus:}

$\triangle \nabla$ Nichterwerbstătig Erwerbstätig $\quad$ Arbeitslos gemeldet

Schaubild 1 Übergänge aus der Arbeitslosigkeit.

der Komplexität und Vielfalt der dabei involvierten Prozesse hinreichend gerecht wird, da sowohl die „subjektive“ Betroffenheit als auch die „objektiven" Folgen von Arbeitslosigkeit trotz gleicher individueller Statuslage je nach der Personenkonstellation im Haushalt durchaus differenzierte Auswirkungen zeitigen können. Es ist anzunehmen, daß sowohl Einkommenseinbußen als auch psychische Belastungen, die durch Arbeitslosigkeit verursacht werden, durch Interventionen einer Haushaltsgemeinschaft in ihren Auswirkungen beeinflußt werden. Mit dem Sozio-ökonomischen $\mathrm{Pa}$ $n e l^{2}$ (SOEP) ist es möglich, einen empirischen Zugang zu einzelnen Aspekten der Arbeitslosigkeit zu eröffnen, der bisher weder mit den Daten der amtlichen Arbeitsmarktstatistik noch mit anderen Umfragedaten möglich war:

- Es sind haushaltsspezifische Typen von Arbeitslosigkeit bzw. von Arbeitslosen unterscheidbar (Krause, 1987).

- Diese Typen zeigen unterschiedliche Verlaufsformen, wenn man sie über die Jahre 1984, 1985 und 1986 betrachtet, was nur mit einer Längsschnitterhebung, wie sie das Panel darstellt, möglich ist.
Man kann die „objektiven" Folgen von Arbeitslosigkeit im Haushaltskontext untersuchen, nämlich Veränderungen des Haushaltseinkommens.

- Es wird möglich, die ,subjektiven" Folgen von Arbeitslosigkeit im Haushaltskontext aufzuzeigen: Zufriedenheiten von Haushaltsvorständen, Ehepartnern und ledigen Kindern, auch in der wechselseitigen Bewertung ihrer Situation.

\section{Typen und Verläufe von Arbeitslosigkeit und Nichterwerbstätigkeit bei Haushaltsvorständen, (Ehe-)partnern und ledigen Kindern}

Betrachtet man Arbeitslosigkeit im Haushaltskontext, so lassen sich drei Typen bzw. drei ${ }^{3}$ unterschiedliche Verläufe von Arbeitslosigkeit identifizieren (Schaubild 1).

Typ 1: Über 60 Prozent der Arbeitslosen des SOEP sind Haushaltsvorstände, größtenteils Männer. Im Zeitablauf verbleiben die meisten von ihnen jedoch nicht lange ohne Arbeit. Bezogen auf den Übergang der 1985 neu arbeitslos gemeldeten Haushaltsvorstände (Gruppe B) zeigt sich, daß über die Hälfte von ihnen ein Jahr später wieder erwerbstätig wurde; ein Drittel ist auch 1986 weiterhin arbeitslos; ein Sechstel scheidet aus der Erwerbstätigkeit aus.

Typ 2: Etwa ein Viertel der Arbeitslosen sind (Ehe-)Partner, meist Frauen. Ihr Verlaufsprozeß zeichnet sich zwar ebenfalls durch eine geringe Verbleibewahrscheinlichkeit im Arbeitslosenstatus

${ }^{2}$ Das Projekt „Das Sozio-ökonomische Panel“ (SOEP) ist ein Teilprojekt des Sonderforschungsbereich 3 ( $\mathrm{Sfb}$ 3) „Mikroanalytische Grundlagen der Gesellschaftspolitik" der Universitäten Frankfurt und Mannheim, das in Zusammenarbeit mit dem Deutschen Institut für Wirtschaftsforschung (DIW, Berlin) durchgeführt wird. Im Rahmen des Projekts wurden ab 1984 jährlich in ca. 6000 Haushalten der Bundesrepublik Deutschland alle Haushaltsmitglieder, die 16 Jahre und älter waren, befragt (Welle $1 \mathrm{ca} .12000$ Personen). Die dabei angesprochenen Themengebiete (Einkommen, Wohnsituation, Zeitverwendung, Gesundheit, Erwerbsbeteiligung, regionale Mobilität, Bildung, Zufriedenheiten, Werteinstellungen usw.) ermöglichen es, ein breites Spektrum von Lebensbereichen im Längsschnitt und auch auf der Haushaltsebene zu untersuchen (Hanefeld 1987).

${ }^{3}$ Die Verlaufstypen anderer arbeitsloser Haushaltsangehöriger sind aufgrund ihrer geringen Fallzahl nicht angeführt. 
aus, jedoch ist bei ihnen zugleich auch die auf den Erwerbsstatus bezogene „Erfolgsquote" am geringsten. Knapp 33 Prozent der 1985 neu arbeitslos gemeldeten (Ehe-)Partner schaffte ein Jahr später die Rückkehr in den Beruf (Gruppe B). Der größte Teil $(37.3 \%)$ zieht sich - zumindest vorläufig aus dem Erwerbsleben zurück; 30 Prozent verbleiben als arbeitslos Gemeldete.

Typ 3: Die kleinste Gruppe bilden die im Haushalt lebenden ledigen Kinder des Haushaltsvorstandes. Obwohl die Fallzahl für eine weiterreichende Interpretation ihres Statusverlaufs zu gering ist, kann festgehalten werden, daß sich gerade für diese sozialpolitisch besonders brisante Gruppe jugendlicher Arbeitsloser ein vergleichsweise schneller Rückstrom bzw. Übergang in das Erwerbsleben abzeichnet.

Die Statusübergänge aller Arbeitslosen in $1984^{4}$ (Gruppe A) ähneln tendenziell bis 1986 in hohem Maße den Verlaufstypen der 1985 neu arbeitslos gemeldeten Personen (Gruppe B). Bei Verläufen von Arbeitslosigkeit sind somit im Haushaltskontext drei Übergangsformen erkennbar, von denen - zumindest auf der deskriptiven Darstellungsebene - die der Haushaltsvorstände Parallelen mit den Verläufen arbeitsloser Jugendlicher aufweisen. Diese Parallelität sollte jedoch nicht über die jeweilige Bedeutung der Betroffenheit von Arbeitslosigkeit hinwegtäuschen: Der Verlust der Erwerbsarbeit des Haushaltsvorstandes bedeutet für den Haushalt meist den Wegfall der Haupteinkommensquelle, für den Haushaltsvorstand selbst auch den Verlust einer mit Sozialprestige versehenen Statusposition. Obwohl Arbeitslosigkeit für die (noch) im Elternhaushalt lebenden Kinder finanziell weniger schwerwiegende Folgen haben kann, ist die Betroffenheit von Arbeitslosigkeit bei Jugendlichen - gerade zu Beginn der Erwerbskarriere - meist eine schmerzliche Erfahrung, die sich sowohl auf den weiteren Berufsverlauf, wie auch auf die Einstellungen Jugendlicher negativ auswirkt ${ }^{5}$.

\footnotetext{
${ }^{4}$ Gruppe A von Schaubild 1 umfaßt nicht exakt denselben Personenkreis wie Gruppe B (Gruppe B: 1985 neu arbeitslos Gemeldete), da sie auch Personen miteinbezieht, die schon vor 1984 arbeitslos gemeldet waren.

${ }^{5} \mathrm{Zu}$ den psycho-sozialen und finanziellen Auswirkungen von Arbeitslosigkeit bei Jugendlichen s. Schober 1987. Das spezielle Problem von Phasen der Arbeitslosigkeit zu Beginn des Erwerbslebens und deren Auswirkungen auf Einstellungen und Erwartungen von Jugendlichen behandelt Habich 1985.
}

Eine andere Verlaufsform von Arbeitslosigkeit zeichnet sich für (Ehe-)Partner ab. Zum Teil erkennbar sind noch die auf traditionellen Berufsorientierungen basierenden Erwerbskarrieren verheirateter Frauen. Nach wie vor ist die Hausfrauenrolle eine gesellschaftlich anerkannte Alternative zur weiblichen Erwerbstätigenrolle. Der hohe Abstrom arbeitsloser Ehefrauen in den Nichterwerbstätigenstatus bei kritischen Arbeitsmarktsituationen wird deshalb insgesamt nicht erstaunen, da aufgrund der - meist durch familiale Gründe verursachten - diskontinuierlichen Erwerbsverläufe von Frauen (Landua, 1986: Kap. 2) diese, besonders bei guten Verdiensten des Ehepartners, häufiger als Männer keine oder nur kurzfristige Leistungen vom Arbeitsamt beziehen und deshalb auch eher davon Abstand nehmen, sich weiter beim Arbeitsamt zu melden. Es sei weiterhin erwähnt, daß die Kontrastierung der Arbeitslosen mit der größeren Gruppe der ,Nichterwerbstätigen" (Hausfrauen, in Ausbildung befindliche Personen usw.) zu dem Ergebnis führt, daß die Muster der Verläufe aller drei nichterwerbstätigen Haushaltsmitgliedergruppen starke Ähnlichkeit mit denen der Arbeitslosen aufweisen. Besonders nichterwerbstätigen Haushaltsvorständen und ledigen Kindern gelingt es vergleichsweise kurzfristig, (wieder) auf dem Arbeitsmarkt in Erscheinung zu treten. Für (Ehe-)Partner von Haushaltsvorständen ist hingegen der Verbleib in der Nichterwerbstätigkeit am häufigsten zu beobachten. Diese Personengruppe bildet das größte Reservoir der sogenannten „Stillen Reserve“ (Landua/Zapf, 1989: $11 \mathrm{ff})$.

\section{Determinanten von Arbeitslosigkeitsverläufen einzelner Haushaltsmitgliedergruppen - Ergebnisse einer LOGIT-Analyse}

Obwohl die identifizierten unterschiedlichen Verlaufstypen von Arbeitslosigkeit der drei Haushaltsmitgliedergruppen sich sehr prägant voneinander abheben, ist zumindest nicht auszuschließen, daß ein Teil dieser Unterschiede auch durch andere Einflußgrößen ,,verursacht" wird. Fragestellungen wie diese lassen sich nur mit Hilfe multivariater Analyseverfahren beantworten. Verwendet wurde hier ein „LOGIT-Modell“, als Spezialfall eines allgemeinen „Log-linearen Modells“ (Fahrmeier/ Hamerle, 1984: Kap. 10). Erklärt werden sollte der ,erfolgreiche“ Abgang aus dem Arbeitslosenstatus zwischen 1984 und 1986. Tabelle 1 enthält 
Tabelle 1 Determinanten der Verläufe von Arbeitslosigkeit, 1984 bis 1986 - Ergebnisse einer LOGITAnalyse.

Verhältnis- Signifikoeffizient kanz

(1) $>0.05$

\section{Responsevariable:}

- „Erfolg" (2) vs....

Faktorvariablen:

- Stellung im Haushalt

- Haushaltsvorstand (HHV)

0.46

- (Ehe-)Partner des HHV

0.16

- Kinder des HHV

0.22

- Alter 1984

- Bis 44 Jahre

0.77

- 45 Jahre und älter

0.17

- Gesundheitliche Behinderung (3)

- Überhaupt nicht

0.51

- Ein wenig, stark

0.26

- (Schulbildung)

- Volks- Hauptschulabschluß

- Mittlere Reife, Abitur

0.38

0.33

n.s.

- Schulbildung* Alter (4)

- Bis 44 Jahre

0.27

- 45 Jahre und älter

0.46

Likelihood Ratio $=13.91 ; D F=15 ; P=0.54$

(1) Um leichter interpretierbare Werte zu erhalten, wurden die LOGIT-Parameter in entlogarythmierte Koeffizienten rücktransformiert (zur Methode vgl. NORUSIS, 1985:334ff).

(2) Responsevariable: $0=$ „Vorjahr: arbeitslos, aktuelles Jahr: erwerbstätig" (Erfolg); 1 = „Vorjahr: arbeitslos, aktuelles Jahr: weiterhin ohne Beschäftigung (arbeitslos, nicht erwerbstätig).

(3) Frage: "Behindert Sie Ihr Gesundheitszustand bei der Erfüllung alltäglicher Aufgaben?".

(4) Bezugskategorie ist "niedriger Schulbildungsabschluß" (Volks-, Hauptschulabschluß).

Datenbasis: Längsschnittdaten des Sozio-ökonomischen Panel; Welle 1 (1984) bis Welle 3 (1986).

die Ergebnisse des getesteten Modells. Was die Frage nach der Gültigkeit des Modells betrifft ergibt sich, daß die Maximum-Likelihood-Statistik mit einem Wert von 0.54 insgesamt für eine sehr gute Modellanpassung spricht. Für die Interpretation der Daten ist weiterhin festzuhalten, daß das (geschätzte) Verhältnis von „erfolgreichen“ und kontinuierlichen Verläufen der Erwerbslosigkeit in der Gesamtpopulation bei 0.36:1 liegt. Dieses
Verhältnis dient für die folgenden Darstellungen als Maßzahl.

Es zeigt sich, daß auch bei Kontrolle anderer Einflußgrößen die "Stellung im Haushalt" sich als die bedeutendste Faktorvariable für die Verläufe von Arbeitslosigkeit erweist: Haushaltsvorständen (0.46: 1) gelingt es weitaus häufiger als dem $\mathrm{Ge}$ samtdurchschnitt, wieder erwerbstätig zu werden. Umgekehrt bleiben (Ehe-)Partner (0.16: 1) mehr als zwei mal so oft weiterhin ohne Arbeit wie alle Personen. Interessanterweise schneiden im multivariaten Verfahren die arbeitslosen Kinder ( 0.22 : 1) insgesamt schlechter ab als die Gesamtpopulation, was vor allem durch die Kontrolle anderer Bestimmungsgrößen zu erklären ist. Hierzu zählt zweifelsohne das Alter der von Arbeitslosigkeit betroffenen Personen: Jüngeren Arbeitslosen (0.77: 1) gelingt es doppelt so häufig wie allen Betroffenen, (erneut) eine Arbeit zu finden. Auch gesundheitliche Beeinträchtigungen (0.26: 1$)$ erweisen sich als signifikante Hemmnisse für eine mögliche Rückkehr in das Erwerbsleben. Der Einfluß der Schulbildung ist nicht unmittelbarer Art, sondern wird über andere Variablen vermittelt. So ist festzuhalten, daß selbst jüngere Arbeitslose, deren weitere Erwerbschancen sich insgesamt als recht gut erwiesen, dann damit rechnen müssen längere Zeit ohne Beschäftigung zu bleiben, wenn sie nur über ,niedrige“ Schulabschlüsse verfügen (0.27: 1).

Bei Anwendung multivariater Verfahren erweist sich demnach einerseits, daß die Verläufe von Arbeitslosigkeit durch eine Reihe von EinflußgröBen bestimmt werden, andererseits, daß auch bei Kontrolle anderer Faktoren die Chance für eine erneute Erwerbstätigkeit in hohem Maße davon abhängig zu sehen ist, welche Stellung eine Person innerhalb des Haushalts einnimmt.

\section{Einkommenseinbußen in Abhängigkeit von haushaltsinternen Erwerbskonstellationen}

Durch Arbeitslosigkeit induzierte Prozesse der Abwärtsmobilität bewirken, neben den psychosozialen Folgen für die betroffenen Personen, insbesondere einkommensbezogene Veränderungen. Neuere Studien gehen dabei über den individuellen Kontext hinaus und betonen, daß die Auswirkungen von Arbeitslosigkeit auf die soziale Stellung und insbesondere die Gefahr der Verarmung sehr stark vom Familien- bzw. vom Haushaltskontext mitgeprägt werden (Zapf et al., 1987: $93 \mathrm{ff}$; 
Tabelle 2 Die Abhängigkeit der Höhe des Haushaltsnettoeinkommens von der Erwerbskonstellation im Haushalt. Ergebnisse einer Multiplen Klassifikationsanalyse - 1985.

\section{Grand Mean: 2947 DM}

N $\quad \begin{gathered}\text { (Unkontrollierte) } \\ \text { Abweichung vom Grand Mean (in DM) }\end{gathered}$

(Kontrollierte) (1)
Abweichung vom Grand Mean (in DM)

eta beta

\section{Erwerbskonstellation}

im Haushalt 1985:

$\begin{array}{rccrrr}\text { HHV } & \text { PAR } & \text { KIN (2) } & & & \\ + & + & +(3) & 479 & 1204 & 1074 \\ + & + & - & 2157 & 561 & 552 \\ + & - & - & 2313 & -107 & -157 \\ + & - & + & 441 & 997 & 865 \\ - & + & + & 6 & 955 & 813 \\ - & - & - & 133 & -1225 & -1280 \\ - & + & - & 75 & -735 & -709 \\ - & - & + & 14 & -1102 & -1203\end{array}$

Einpers.haushalte (HHV):

/ Voll enwerbstätig

/ Teilzeiterwerbstätig

/ Arbeitslos gemeldet

/ Nichterwerbstätig

$\begin{array}{rrr}469 & -937 & -669 \\ 46 & -1802 & -1563 \\ 51 & -2110 & -1868 \\ 714 & -1545 & -1361\end{array}$

$\mathrm{R}_{2}=.49$

$\mathrm{R}=.24$

Signifikanz $=0.000$

(1) Als Kovariaten gehen "Alter" und "Haushaltsgröße" in das Modell ein.

(2) HHV = Haushaltsvorstand; PAR = (Ehe-)Partner; KIN = Kinder des HHV.

(3) $"+"$ = Erwerbstätig; ",- = Arbeitslos gemeldet.

Datenbasis: Sozio-ökonomisches Panel; Gewichtete Längsschnittdaten von Welle 1 (1984) bis Welle 3 (1986).

Klein, 1987a). Deshalb ist die Bedeutung von Haushaltsgemeinschaften für die einkommensbezogenen Auswirkungen ${ }^{6}$ von Arbeitslosigkeit spezieller Gegenstand dieses Kapitels.

Tabelle 2 weist darauf hin, daß die Folgen der Betroffenheit von Arbeitslosigkeit in hohem Maße davon abhängig zu sehen sind, welche Einkommensquellen für den Haushalt ausfallen. Besonders gravierend wirken sich die „Verluste“ von Hauptverdienern (Haushaltsvorstände, z. T. auch Kinder) oder mehreren Verdienern im Haushalt aus. Vergleichsweise geringere Einkommenseinbußen entstehen hingegen durch die Arbeitslosigkeit des (Ehe-)Parnters. Es wäre jedoch voreilig, die hierdurch verursachten finanziellen Folgen all-

\footnotetext{
${ }^{6}$ Beachtung finden dabei allerdings reale Veränderungen im Einkommensniveau des Haushaltsnettoeinkommens und nicht Veränderungen bspw. eines bedarfsgewichteten Haushalts-Pro-Kopf-Einkommens (s. hierzu Zapf et al. 1987: $93 \mathrm{ff}$ ) das sich als Variable besser zur Messung des Zusammenhangs von Massenarbeitslosigkeit und Einkommensarmut eignet.
}

gemein zu verharmlosen. Gerade in einkommensschwachen Haushalten ist der Einkommensbeitrag, den erwerbstätige Ehefrauen leisten, häufig fast ebenso hoch wie der des Haushaltsvorstands. Durch die Arbeitslosigkeit des Ehepartners bedingte Einkommensdefizite zeigen in diesem Fall sehr starke Auswirkungen auf die finanzielle Lage von Haushalten (Krause, 1987: 112).

Obwohl hier die realen Veränderungen des Haushaltsnettoeinkommens betrachtet werden, gibt es auch Indizien, die einen Zusammenhang zwischen der Familiengröße bzw. -zusammensetzung sowie der Verdienerzahl im Haushalt und der Gefahr der Verarmung durch Arbeitslosigkeit zeigen. Selbst der Ausfall des Haushaltvorstandes als Erwerbsperson in Haushalten mit zwei oder mehr Einkommensbeziehern kann durch die verbleibenden Einkommensquellen in seiner Tragweite wenigstens teilweise aufgefangen werden ${ }^{7}$. Nicht unerwähnt

${ }^{7}$ Zur Auffangwirkung anderer Faktoren wie bspw. einkommensunabhängige Sozialleistungen s. Klein 1987b; sowie Büchtemann 1985. 
bleiben darf allerdings, daß diese Form von haushaltsbezogener Absicherung vor materiellen Folgen der Arbeitslosigkeit nur vergleichsweise wenigen Haushalten vorbehalten ist.

\section{Der Einfluß diskontinuierlicher Erwerbsverläufe auf haushaltsinterne Veränderungen der allgemeinen Lebenszufriedenheit}

Lebensqualität oder individuelle Wohlfahrt konstituiert sich nach unserem Verständnis nicht nur aufgrund „objektiver“ Lebensbedingungen - im Sinne von (im Prinzip) beobachtbaren Lebensverhältnissen wie dem Haushaltseinkommen -, sondern auch durch die von den Betroffenen selbst abgegebenen Einschätzungen ihrer Lebensbedingungen. Unter solchen Einschätzungen sind einerseits Zufriedenheitsangaben, aber andererseits auch generellere kognitive Gehalte wie Hoffnungen, Ängste, Sorgen usw. zu verstehen. Nach den Auswirkungen der Arbeitslosigkeit auf die objektiven Lebensbedingungen von betroffenen Personen soll im folgenden untersucht werden, ob sich erkennbare Reaktionen auch auf der Ebene subjektiver Wohlfahrtskomponenten widerspiegeln.

Beachtung finden dabei Veränderungen der allgemeinen Lebenszufriedenheit ${ }^{8}$ - als Maß der Gesamtbewertung der Lebensumstände einer Person. Die negativen Folgen des Verlustes von zentralen sinnstiftenden Lebenskomponenten für das Individuum lassen vermuten, daß der Verlust der Erwerbsarbeit subjektiv als erhebliche Einschränkung der Lebensqualität bewertet wird.

Schaubild 2 zeigt für mehrere Teilgruppen die durchschnittliche Lebenszufriedenheit für die Jahre 1984 bis 1986. Auf der Ebene aller Befragten (A) reflektiert sich das bekannte hohe und im Aggregat stabile Niveau der Lebenszufriedenheit in der Bundesrepublik. Nennenswerte Abweichungen von diesem Muster sind für alle Erwerbstätigen (B), aber auch für die kontinuierlich erwerbstätigen Haushaltsmitgliedergruppen (C D E) nicht erkennbar. Teilgruppe F schließt jene Erwerbstätigen ein, die von 1984 auf 85 arbeitslos und von 1985 auf 86 wieder erwerbstätig wurden. Die Interpretation der durchschnittlichen Lebenszufrieden-

${ }^{8}$ Frage: „Wie zufrieden sind Sie gegenwärtig, alles in allem, mit ihrem Leben?“ 11 -stufige Skala von 0 bis 10 ; $0=$,ganz und gar unzufrieden“ $10=$,ganz und gar zufrieden“.

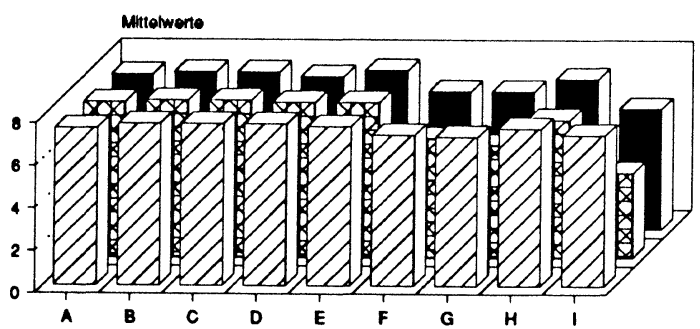

Teilgruppen

Zutnodenhait 1984 $\quad[D$ Zutriodonhoit 1985

Zutriedonhert 1086

Datenbasis: Sozio-ökonomisches Panel -

Gewichtete Längsschnittdaten 1984-86.

Teilgruppen:

Durchschnittliche Lebenszufriedenheit von:

A - Allen Befragten.

B - Allen Enwerbstätigen (erwerbstätig von 1984 bis 1986).

C - Allen erwerbstätigen Haushaltsvorständen (erwerbstätig von 1984 bis 1986).

D - Allen erwerbstätigen (Ehe-)Partnern von Haushaltsvorständen (erwerbstätig von 1984 bis 1986).

E - Allen erwerbstätigen Kindern von Haushaltsvorständen (erwerbstätig von 1984 bis 1986).

F - Allen Befragten; 1984: erwerbstätig, 1985 arbeitslos, 1986: erwerbstätig.

G - Haushaltsvorständen; 1984: erwerbstätig, 1985: arbeitslos, 1986: enwerbstätig.

H - (Ehe-)Partnern von Haushaltsvorständen; 1984: erwerbstätig, 1985: arbeitslos, 1986: erwerbstätig.

1 - Kindern von Haushaltsvorständen; 1984: erwerbstätig, 1985: arbeitslos, 1986: erwerbstätig.

Schaubild 2 Lebenszufriedenheit im Zeitablauf - 1984 bis 1986.

heiten im jeweiligen Jahr legt eindeutig den Schluß nahe, da $B$ Arbeitslosigkeit einen erheblichen Verlust an subjektivem Wohlbefinden der Betroffenen zur Folge hat. Betrachtet man den gleichen Sachverhalt wiederum differenziert nach den einzelnen Haushaltsmitgliedern, so zeigen sich jedoch erneut bermerkenswerte Unterschiede: Arbeitslosigkeit wirkt sich bei den hier berücksichtigten Übergängen unmittelbar vor allem auf die Zufriedenheiten von Haushaltsvorständen und noch stärker auf die der Kinder im Haushalt aus. Eindeutig folgen hier die Veränderungen der Zufriedenheitswerte dem Wandel des Erwerbsstatus. Weniger eindeutige Aussagen hingegen lassen sich für (Ehe-)Partner 
machen. Ihre Lebenszufriedenheit wird durch Arbeitslosigkeit bedingte Erwerbsunterbrechungen im Vergleich zu den anderen Haushaltsmitgliedern nur geringfügig negativ beeinflußt. Offensichtlich intervenieren in die Lebenszufriedenheit von Frauen teilweise noch in erheblichem Umfang Faktoren bzw. Lebensbereiche, die nicht in unmittelbarem Zusammenhang mit ihrer Erwerbskarriere stehen. $\mathrm{Ob}$ dieses Phänomen möglicherweise aber auch auf unterschiedliche Attributionsprozesse der Ursachen der eigenen Arbeitslosigkeit bei Männern und Frauen zurückzuführen ist, steht noch zur Klärung offen. Eine Antwort erscheint mehr als wünchenswert, da zum einen zweifelsohne die individuelle Belastung durch Arbeitslosigkeit in hohem Maße von der subjektiven Ursachenzuschreibung abhängig ist und zum anderen ein enger Zusammenhang zwischen der Erfolgswahrscheinlichkeit bei der Suche nach einem neuen Arbeitsplatz und der Ursachenattribuierung der eigenen Arbeitslosigkeit zu vermuten ist (Kirchler, 1984; Grau/Thomsen, 1985; allgemein: Kelley, 1972a und 1972b).

\section{Arbeitslosigkeit und die wechselseitige Veränderung der Lebenszufriedenheit von Haushaltsvorständen und deren (Ehe-)Partnern im Haushaltskontext}

Versteht man den Haushalt bzw. die Familie nicht nur als einen „Ort" des Zusammenlebens, sondern auch als eine Gemeinschaft, in der psychische Belastungen direkt mit anderen Personen geteilt und verallgemeinert werden, so wäre zu erwarten, daß einschneidende Veränderungen im Leben einer Person dieser Gemeinschaft sich auch auf das Lebensumfeld der anderen Haushaltsmitglieder auswirken. Tabelle 3 verweist hierbei auf ein interessantes Muster. Zunächst zeigt sich - gemäß den vorangegangenen Erläuterungen, da $\beta$ Haushaltsvorstände deutlich stärker von statusbedingten Veränderungen der subjektiven Wohlfahrt betroffen sind als ihre (Ehe-)Partner, wenn sie selbst arbeitslos, bzw. wieder erwerbstätig werden. Die Differenz beträgt $-1.3(+1.5)$ Punkte auf einer Elferskala gegenüber $-/+0.5$ bei den (Ehe-) Partnern. Aber (Ehe-)Partner nehmen - bei konstantem eigenem Berufsstatus - viel stärkeren Anteil an Veränderungen des Erwerbsstatus „ihres“ Haushaltsvorstandes, als dies umgekehrt der Fall ist. Dies sollte nun nicht vorschnell dahingehend interpretiert werden, daß (die zumeist männlichen) Haushaltsvorstände weniger „Sensibilität“
Tabelle 3 Veränderungen der Lebenszufriedenheit im Haushaltskontext - Ergebnisse im Rahmen individueller Veränderungen des Erwerbsstatus (1).

Aktuelle Lebenszufriedenheit (2)

HHV (Ehe-)Partner

1985198619851986

Mean (Differenzen in Klammer)

Statusübergänge (1985 bis 86 ):

- Haushaltsvorstand (3)

- Erwerbst.>arbeitslos

$(-1.3)$

$(-0.4)$

$\begin{array}{llll}7.2 & 5.9 & 7.0 & 6.6\end{array}$

- Arbeitsios> Erwerbst.

$(+1.5)$

$(+0.8)$

$\begin{array}{llll}4.9 & 6.4 & 5.8 & 6.6\end{array}$

- (Ehe-)Partner (4)

- Erwerbst.>arbeitslos

$(-0.2)$

$\begin{array}{llll}7.5 & 7.3 & 7.2 & 6.7\end{array}$

$(-0.1)$

$(+0.5)$

- Arbeitsios >erwerbst.

$\begin{array}{ll}7.3 & 7.2\end{array}$

6.67 .1

- Status HHV und (Ehe-)-

$\begin{array}{ll}7.4 & 7.4\end{array}$

$7.5 \quad 7.4$

Partner ohne Veränderung

(1) Haushaltsgröße und Erwerbsstatus der Kinder im Haushalt werden kontrolliert.

(2) Durchschnittswerte auf einer 11-stufigen Skala; $0=$ "ganz und gar unzufrieden", $10=$ "ganz und gar zufrieden".

(3) Der Erwerbsstatus des (Ehe-)Partners bleibt konstant.

(4) Der Erwerbsstatus des Haushaltsvorstands bleibt konstant.

Datenbasis: Sozio-ökonomisches Panel; gewichtete Längsschnittdaten von Welle 1 (1984) bis Welle 3 (1986).

oder „Empathie“ gegenüber dem Schicksal ihres Partners aufbringen, denn dieses unterschiedliche Zufriedenheitsgefälle könnte auch die Folge unterschiedlicher „objektiver" Veränderungen im Haushalt sein: Die Arbeitslosigkeit des Haushaltsvorstandes zeigt - wie erwähnt (s.o.) - deutlich stärkere Auswirkungen auf die Höhe des Haushaltsnettoeinkommens als die Arbeitslosigkeit von (Ehe-)Partnern. Einkommensveränderungen betreffen jedoch i.d. R. alle Haushaltsmitglieder, so daß eine Beziehung zwischen „objektiven“ und „subjektiven" Variablen in der vorgefundenen Art bei konstantem eigenen Erwerbsstatus durchaus plausibel erscheint.

Generell kann Arbeitslosigkeit bezüglich ihrer subjektiven Folgen also nicht ausschließlich als individuelles Schicksal begriffen und verstanden werden. Betroffenheit zeichnet sich auch bei Haus- 
haltsmitgliedern ab, die persönlich von den Folgen des Verlusts ihrer Erwerbsarbeit unberührt bleiben. Mit einer rein auf das Individuum bezogenen Arbeitslosenstatistik werden deshalb implizit wichtige durch Arbeitslosigkeit induzierte Wirkungen auf das subjektive Wohlbefinden von in Mehrpersonenhaushalten lebenden Personen unterschätzt.

\section{Zusammenfassung und politische Schlußfolgerungen}

Die Konzentration auf eine kontextbezogene Analyse von Arbeitslosigkeit weist gegenüber „klassischen" individualistischen Ansätzen bedeutsame Vorteile auf. Sie ermöglicht insbesondere die Abkehr von einer atomisierten Betrachtung des einzelnen d. $h$. von einer Sichtweise, die individuelle Lebensereignisse ohne den sie umgebenden sozialen Kontext erfaßt. Arbeitslosigkeit ausschließlich als Schicksal der betroffenen Personen aufzufassen, hieße der Komplexität des Phänomens ungenügend Rechnung zu tragen. Ein zentrales Anliegen dieses Beitrages war es deshalb, auf Zusammenhänge hinzuweisen, die erst auf der Basis von Mikrokollektiven als Untersuchungseinheiten sichtbar werden.

Die Kritik an den veröffentlichten Arbeitslosenstatistiken ist meines Erachtens nach somit nicht ganz unberechtigt. Einwände lassen sich aus verschiedenen Richtungen heraus formulieren: So beinhalten offizielle Statistiken einerseits selten Informationen über unterschiedliche Typen von Arbeitslosigkeit mit unterschiedlichen Verläufen und unterschiedlichen objektiven und subjektiven Folgen für die Betroffenen und/oder - im Haushaltskontext - für deren Angehörige. Aus diesem Defizit folgt jedoch nicht, daß die Arbeitslosenzahlen „zu hoch“ sind und Arbeitslose nur noch dann in der Statistik erfaßt werden sollten, „... wenn sie dem Arbeitsmarkt wirklich zur Verfügung stehen ..." oder eine Arbeitsstelle suchen, ,... . die für sie die einzige oder eine wesentliche Einkommensquelle darstellt" (zit. nach Tagesspiegel, 1988: 19/5). Durchaus kann aber daraus gefolgert werden, daß Arbeitslosigkeit kein Einheitsphänomen ist, sondern nach bestimmten Typen bzw. Verlaufsformen unterschieden werden sollte. Andererseits lassen sich auch Einwände in Richtung einer Unterschätzung des Problems der Arbeitslosigkeit durch die amtlichen Veröffentlichungen formulieren: Wenig Informationen liegen bspw. über die sogenannte "Stille Reserve" vor, also über das Potential an Arbeitskräften, das nicht über das Arbeitsamt Zu- gang zur öffentlichen Statistik von Arbeitssuchenden findet; und wenig Kenntnisse existieren darüber, wie viele Personen in der Lage sind, sich bietende Opportunitäten am Arbeitsmarkt - zum Teil entgegen den eigenen bis dahin bestehenden Berufsplänen - wahrzunehmen.

Mikroanalysen auf der Ebene von Betroffenen in ihrem Haushaltskontext lösen keine Probleme des Arbeitsangebots und der Arbeitsnachfrage auf der gesamtwirtschaftlichen Ebene, wie sie etwa durch wirtschaftlichen Strukturwandel oder durch den Berufseintritt der geburtenstarken Jahrgänge entstehen. Sie ergeben aber neue Einsichten in die Umstände und Folgen von Arbeitslosigkeit sowie in die Erwartungen und Handlungen von Nichterwerbstätigen gegenüber dem Arbeitsmarkt. Diese Einsichten sollten auch für die Arbeitsmarktpolitik von Interesse sein.

\section{Literatur}

Brandes, W./Weise, P. 1980: „Arbeitsmarkt und Arbeitslosigkeit “; Physica-Verlag, Würzburg/Wien.

Büchtemann, Ch. F. 1985: „Soziale Sicherung bei Arbeitslosigkeit und Sozialhilfebedürftigkeit"; in: Mitteilungen aus der Arbeitsmarkt- und Berufsforschung, H. 4, S. 450-466.

Büssing, A. 1987: „Arbeitsplatzunsicherheit und Antizipation von Arbeitslosigkeit als Stadien des Arbeitslosigkeitsprozesses“; in: Soziale Welt, H. 3, S. 309-329.

Fahrmeier, L., Hamerle, A., (Hrsg.), 1984: „Multivariate statistische Verfahren", Berlin/New York.

Franke, H./Prast, F. 1985: „Arbeitslosigkeit - Fakten, Ursachen, Lösungsansätze“; in: Spektrum der Wissenschaft, H. 9, Sept. 85, S. 32-47.

Globus Kartendienst GmbH 1988: „Globus-Zahlenbilder", Nr. Ka-7389, 43. Jg., Hamburg.

Grau, Th./Thomsen, K. 1985: „Die Attribuierung des Vorwurfs der Arbeitsunwilligkeit - Zur Rolle der Arbeitslosen"; in: Th. Kieselbach/A. Wacker (Hrsg.), ,Individuelle und gesellschaftliche Kosten der Massenarbeitslosigkeit‘, Beltz Verlag, Weinheim/Basel, S. 107-119.

Habich, R. 1985: „Arbeitslosigkeit zu Beginn des Erwerbslebens: Betroffenheit und Auswirkungen auf Berufsverlauf und Einstellungen"; in: M. Kaiser et al. (Hrsg.), ,Berufliche Verbleibsforschung in der Diskussion', Beiträge zur Arbeitsmarkt- und Berufsforschung, 90.1, Nürnberg, S. 575-595.

Hanefeld, U. 1987: „Das Sozio-ökonomische Panel. Grundlagen und Konzeption"; Campus-Verlag, Frankfurt a. M./New York.

Hofbauer, H./Dadzio, W. 1984: „Berufliche Weiterbildung für Arbeitslose", in: MittAB, H. 2, S. 183-200.

Institut für Arbeitsmarkt- und Berufsforschung (Hrsg.) 1988: „Zahlen-Fibel“, Ausgabe 1988, Nürnberg. 
Kelley, H. H. 1972a: „Attribution in Social Interaction“; in: E. E. Jones et al. (Hrsg.), ,Attribution: Perceiving the causes of behavior', General Learning Press, Morristown/N. Y., S. 1-26.

Kelley, H. H. 1972b: „Causal Schemata and the Attribution Process"; in E. E. Jones et al. (Hrsg.), ,Attribution: Perceiving the causes of behavior', General Learning Press, Morristown/N. Y., S. 151-174.

Kieselbach, Th./Wacker, A. (Hrsg.): „Individuelle und gesellschaftliche Kosten der Massenarbeitslosigkeit"; Beltz Verlag, Weinheim/Basel.

Kirchler, E. 1984: „Arbeitslosigkeit und Alltagsbefinden. Eine sozialpyschologische Studie über die subjektiven Folgen von Arbeitslosigkeit"; Universitätsverlag R. Trauner, Linz.

Klein, Th. 1987a: „Familiale Verarmung durch Arbeitslosigkeit"; in: Kölner Zeitschrift für Soziologie und Sozialpsychologie, Jg. 39, S. 534-549.

Klein, Th. 1987b: „Arbeitslosengeld, Arbeitslosenhilfe und Wohngeld im Kampf gegen Verarmung und Abstieg bei Arbeitslosigkeit"; in: MittAB, H. 3, S. $351-367$.

Krause, P. 1987: „Lebensbedingungen und wahrgenommene Lebensqualität von Arbeitslosen"; in: H.-J. Krupp/U. Hanefeld (Hrsg.), ,Lebenslagen im Wandel: Analysen $1987^{\circ}$, Campus Verlag, Frankfurt a. M./New York, S. 105-125.

Kreutz, H./Fröhlich, G./Maly, D. 1984: „Alternative Projekte: Realistische Alternativen zur Arbeitslosigkeit?"; in: MittAB, H. 2, S. 267-273.
Landua, D. 1986: „Determinanten der Unterbrechung und Wiederaufnahme der Erwerbstätigkeit. Eine soziologische Sekundäranalyse mit Paneldaten"; unveröffentl. Diplomarbeit, Lehrstuhl für Soziologie III, Fakultät für Sozialwissenschaften, Universität Mannheim.

Landua, D./Zapf, W., 1989: „Folgen von Arbeitslosigkeit für die Wohlfahrt von Individuen und Haushalten", Arbeitspapier P89-102, Wissenschaftszentrum Berlin. Berlin.

Lederer, E. 1981: „Technischer Fortschritt und Arbeitslosigkeit"; Europäische Verlagsanstalt, Frankfurt a. M.

Rothe, K. 1983: „Arbeitslosigkeit als politische Herausforderung“; Colloquium Verlag, Berlin.

Schober, K. 1987: „Die soziale und psychische Lage arbeitsloser Jugendlicher"; in: MittAB, H. 4, S. 453-478.

Statistisches Bundesamt (Hrsg.) 1985: „Datenreport 1985“, Bundeszentrale für politische Bildung, Bonn.

Der Tagesspiegel 1988: „Unionspolitiker verlangen andere Arbeitslosenstatistik “; in: ,Der Tagesspiegel', unabhängige Berliner Morgenzeitung 19/5.

Townsend, P. 1979: „Poverty in the United Kingdom. A Survey of Household Resources and Standards of Living"; Harmondsworth.

Zapf, W./Breuer, S./Hampel, J./Krause, P./Mohr, H.-M./ Wiegand, E. 1987: „Individualisierung und Sicherheit. Untersuchungen zur Lebensqualität in der Bundesrepublik Deutschland"; Beck Verlag, München. 